\title{
Peculiarities of the Abundances of Neutron-Capture Elements in Galactic Open Clusters
}

\author{
V. A. Marsakov, M. L. Gozha, V. V. Koval', and L. V. Shpigel' \\ Southern Federal University, Rostov-on-Don, Russia \\ e-mail: marsakov@sfedu.ru,gozha_marina@mail.ru,vvkoval@sfedu.ru \\ accepted 2016, Astronomy Reports, Vol. 60, No. 1, pp. 61-72
}

\begin{abstract}
The properties of the relative abundances of rapid and slow neutron-capture elements are studied using a catalog containing spectroscopic abundance determinations for 14 elements produced in various nuclear-synthesis processes for 90 open clusters. The catalog also contains the positions, ages, velocities, and elements of the Galactic orbits of the clusters. The relative abundances of both $r$-elements $(\mathrm{Eu})$ and $s$-elements ( $\mathrm{Y}, \mathrm{Ba}, \mathrm{La}$, and $\mathrm{Ce}$ ) in clusters with high, elongated orbits and in field stars of the Galactic thin disk display different dependences on metallicity, age, Galactocentric distance, and the elements of the Galactic orbits, supporting the view that these objects have different natures. In young clusters, not only barium, but also the three other studied $s$-elements display significantly higher relative abundances than field stars of the same metallicity. The relative abundances of Eu are lower in high-metallicity clusters $([\mathrm{Fe} / \mathrm{H}]>-0.1)$ with high, elongated orbits than in field giants, on average, while the $[\mathrm{Eu} / \mathrm{Fe}]$ ratios in lowermetallicity clusters are the same as those in field stars, on average, although with a large scatter. The metallicity dependence of the $[\mathrm{O}, \mathrm{Mg} / \mathrm{Eu}]$ ratios in clusters with high, elongated orbits and in field stars are substantially different. These and other described properties of the Eu abundances, together with the properties of the abundances of primary $\alpha$-elements, can be understood in a natural way if clusters with high, elongated orbits with different metallicities formed as a result of interactions of two types of high-velocity clouds with the interstellar medium of the Galactic disk: low-metallicity high-velocity clouds that formed from "primordial" gas, and high-metallicity clouds with intermediate velocities that formed in "Galactic fountains".
\end{abstract}

open star clusters, chemical composition, kinematics, Galaxy (Milky Way).

\section{Introduction}

This paper continues our systematic study of the chemical compositions of open clusters, motivated by the existence of clusters with anomalously elongated orbits extending high above the Galactic plane or with low metallicities, which are most likely characteristic of old Galactic subsystems. One proposed explanation for these properties is that the population of open clusters is not uniform, and includes clusters formed partially from interstellar material that fell in from outer volumes of the Galaxy [1-4] (see also references therein). The chemical compositions of such clusters should deviate from those for field stars of the thin disk. To provide a basis for studies of the chemical and spatialkinematic properties of samples of clusters, we have compiled a catalog of 346 clusters based on data published over the past 23 years, including the positions, metallicities, ages, and relative abundances of 14 elements for 90 open clusters, for 82 of which the elements of their Galactic orbits have been calculated [5, Table 2] 1$]$

Our analysis of the relative abundances of $\alpha$ elements in clusters with various kinematic characteristics in [5] supported the existence of different types of clusters with different natures. In particular,

\footnotetext{
${ }^{1} \mathrm{~A}$ full version of this table is available in electronic form at the site ftp://cdsarc.u-strasbg.fr/pub/cats/J/AZh
} 
we found that the relative abundances of primary $\alpha$-elements $([\mathrm{O}, \mathrm{Mg} / \mathrm{Fe}])$ in clusters with high, elongated orbits and field stars of the thin disk display different dependences on metallicity, age, Galactocentric distance, and distance from the Galactic plane. We believe that the observed differences confirm that open clusters with high, elongated orbits and field stars of the thin disk have different natures.

The conclusion of [2] that some clusters formed as a result of interactions of high-velocity, metalpoor clouds with the interstellar material of the Galactic thin disk is also supported by the results of [5]. The relative abundances of $\alpha$-elements for clusters with high, elongated orbits and metallicities $[\mathrm{Fe} / \mathrm{H}]<-0.1$ were found to be lower than the typical values for field stars. This can be explained if the high-velocity clouds that gave rise to them were formed of interstellar material from regions where the star-formation rate and/or the masses of Type II supernovae were lower than near the Galactic plane. On the other hand, the higher relative abundances of primary $\alpha$-elements observed for higher-metallicity clusters with high, elongated orbits, compared to those for field stars, suggests that they formed as a result of interactions between the interstellar medium of the Galactic disk and metal-rich clouds having intermediate velocities, which are currently believed to form from returning gas in so-called "Galactic fountains".

Some studies have shown that open clusters and field stars also have different relative abundances of neutron-capture elements. For example, D'Orazi et al. [6] and Maiorca et al. [7] found that, in contrast to field stars, the relative abundances of slow neutron-capture elements ( $s$-elements) $[s / \mathrm{Fe}]$ in about 20 clusters increase with decreasing age. This could come about due to an increased contribution to enrichment of the interstellar medium by low-mass $\left(<1.5 M_{\odot}\right)$ giants on the asymptotic branch with time [8]. This result is confirmed Mishenina et al. [9, 10], who found an appreciable excess of the relative barium abundances in several young open clusters, while there was no excess in the relative abundances of the other two $s$-elements yttrium and lanthanum.

Most studies have found clusters to have not only barium relative abundance excesses compared to field stars, but also an increase in $[\mathrm{Ba} / \mathrm{Fe}]$ with decreasing age, while no age dependence (see, e.g., [11]), or even a slight growth in their relative abundances with age (see, e.g., [12]), is observed for other $s$-elements. D'Orazi et al. [13] also found appreciable relative barium excesses in three young associations (which also contain open clusters), while other $s$-elements displayed approximately solar $[s / \mathrm{Fe}]$ values. However, it turns out that some field stars - Cepheids - also display excesses of the relative abundances of $s$-elements [14], with the highest excesses being those for barium.

Here, we continue our comparative analysis of the properties of the relative elemental abundances of open clusters and field stars of the thin Galactic disk, and also of different groups of cluster populations, this time looking at rapid $(r)$ and slow $(s)$ neutron-capture elements. Our catalog contains data on the chemical compositions of 90 open clusters taken from 109 primary references, a list of which is provided. The catalog includes relative abundances of both $s$ - (Y, Ba, La, Ce, Nd, and $\mathrm{Zr})$ and $r-(\mathrm{Eu})$ elements. Yttrium abundances are included for 59, zirconium for 52, neodymium for 29, cerium for 38, barium for 73, lanthanum for 55, and europium for 47 clusters. The mean number of studied stars in each cluster is seven. The abundances were determined using only one star in 16 cases; in only five clusters was there only one determination, and [el/Fe] values were available in more than one study in the other cases. For clusters with two or more abundance determinations for a given element, we calculated the mean weighted abundance (see [5] for more detail). Inspection of the $[\mathrm{el} / \mathrm{Fe}]$ values from different studies indicated that the scatter of the values for barium, lanthanum, and europium was $\sigma[\mathrm{el} / \mathrm{Fe}]=0.12 \pm 0.02$, while the scatter for yttrium and cerium was somewhat smaller [5, Table 1]. For comparison, we consulted catalogs of abundances for these same elements in 212 dwarfs, 171 red giants, and 221 field Cepheids of the Galactic thin disk. We also calculated the ages and elements of the Galactic orbits for the red giants. More detail about the parameters of these comparison stars can be found in $[5,14]$. 


\section{METALLICITY DEPENDENCES OF THE RELATIVE ABUN- DANCES OF NEUTRON-CAPTURE ELEMENTS}

$s$-Elements. According to theoretical computations, most of these elements are produced in the cores of giants on the asymptotic branch with masses $M<4 M_{\odot}$, and are then ejected into the interstellar medium in later stages of the star's evolution [15]. The peak production of these elements is at $[\mathrm{Fe} / \mathrm{H}]<-0.2$, and $[s / \mathrm{Fe}]$ decreases with further increase in the metallicity. In addition, some quantity of $s$-element nuclei are produced in the cores of massive stars $\left(M>8 M_{\odot}\right)[16,17]$. (For more detail on the sources of neutron-capture elements, see [14] and references therein.) Since $s$-elements are synthesized in stars with the same masses as Type Ia supernovae, which eject iron-group elements when they explode, the interstellar medium is enriched in both types of elements simultaneously. This means that variations in the relative abundances of $s$-elements with increasing metallicity in a stellar-gas system could be due exclusively to properties of the stars producing them.

Figures 1a-1d present the metallicity dependences of the relative abundances of four $s$-elements (Y, Ba, La, and $\mathrm{Ce}$ ) for open clusters and field giants of the thin disk. Although the theoretical dependence of the output of light $s$-elements $(\mathrm{Y})$ on the metallicity of the stars in which they are synthesized is somewhat different than the corresponding dependence for heavy $s$-elements (Ba, La, $\mathrm{Ce}$ ), we included $\mathrm{Y}$ in this analysis, since the relative abundances of this element were used in [9] in a comparison with the behavior of the barium abundance in young open clusters. Fig. 1a shows that the relative abundances of yttrium display appreciable scatter, for both the clusters and field stars. In order to avoid introducing distortions associated with the theoretical predictions of the outputs of the various elements, we constructed linear regression fits for all the $s$-elements for the range $[\mathrm{Fe} / \mathrm{H}]>-0.2$, where, in fact, most open clusters and field stars of the thin disk are located. The slope of the dependence $d[\mathrm{Y} / \mathrm{Fe}] / d[\mathrm{Fe} / \mathrm{H}]$ for the clusters is equal to zero within $2 \sigma$, while this dependence for the field giants differs from zero by more than $3 \sigma$ (correlation coefficients $r=-0.20 \pm 0.10$ and $r=-0.29 \pm 0.11$, with the probabilities of this correlation arising by chance being $P_{N} \approx 20 \%$ and $\ll 1 \%$, respectively). Although the slopes for the two types of objects coincide within the errors, the mean relative abundances for yttrium for the clusters are higher than those for the field stars by $\Delta\langle[\mathrm{Y} / \mathrm{Fe}]\rangle=0.13 \pm 0.03$

Figure $1 \mathrm{~b}$ presents analogous diagrams for barium. The scatter in the relative abundances of barium is much greater for the clusters than for the field giants, but the correlation for the clusters is significant $\left(r=-0.36 \pm 0.22\right.$, with $\left.P_{N}<1 \%\right)$. Although the regression slopes for the clusters are a factor of 1.5 lower than those for the field stars $(d[\mathrm{Ba} / \mathrm{Fe}] / d[\mathrm{Fe} / \mathrm{H}]=-0.66 \pm 0.24$ and $-0.98 \pm 0.09$, respectively), these two slopes are not distinguishable due to the large uncertainties. At the same time, the mean excess of the relative barium abundances for clusters compared to field giants is very high, $\Delta\langle[\mathrm{Ba} / \mathrm{Fe}]\rangle=0.30 \pm 0.04$. It was concluded in [9], where a similar enhancemnet of $[\mathrm{Ba} / \mathrm{Fe}]$ was found for eight distant and mainly young open clusters, that this enhancement is real, and is not due to uncertainties in the abundances. The same conclusion was drawn in [13].

This excess is equally large for lanthanum, $\Delta\langle[\mathrm{La} / \mathrm{Fe}]\rangle=0.28 \pm 0.03$. The $[\mathrm{La} / \mathrm{Fe}]$ values for clusters and field giants display significant correlations with metallicity, with the same slopes (see Fig. 1c). Note that the scatter between the relative abundances of this element determined in different studies is fairly high, although the increase in the $[\mathrm{La} / \mathrm{Fe}]$ values for clusters compared to field stars exceeds this uncertainty by more than a factor of two; i. e., it is statistically significant. The relative cerium abundances in Fig. 1d for clusters display a very small scatter, and form a narrow sequence in which a break near $[\mathrm{Fe} / \mathrm{H}] \approx-0.2$ can even be seen. This behavior is due to the small uncertainty in the abundances of this element [5, Table 1]. The slopes of the dependences for the clusters and field stars are the same within the uncertainties, with the excess corresponding to $\Delta\langle[\mathrm{Ce} / \mathrm{Fe}]\rangle=0.21 \pm 0.03$.

Thus, the mean relative abundances for all $s$-elements for any metallicity proved to be significantly higher in clusters than in field giants. The absence of significant systematic differences in the abundances of the studied elements for dwarfs and giants is demonstrated by the light gray circles in the figure panels, representing data for field dwarfs. (The regression fit for the dwarfs is not shown 
to avoid cluttering the figure.) In spite of the fact that the regions occupied by the field dwarfs and giants do not always fully coincide, we can see here an enhancement of the relative abundances of all the studied $s$-elements for clusters compared to those for field dwarfs, for a specified metallicity.

$r$-Elements. According to our current understanding, most $r$-elements are synthesized directly during Type II supernova with masses $8<M / M_{\odot}<10$ (see, e.g., [16]). Some quantity of iron-peak elements is also produced in Type II supernova outbursts. Most iron-group elements are produced in Type Ia supsernovae, which represent the final stage in the evolution of close binary stars with masses of $<8 M_{\odot}$ (see, e.g., [18]). Due to the difference in the evolution times of the stars giving rise to these two types of supernovae, the relative abundances $[r / \mathrm{Fe}]$ in subsequent generations of stars should decrease with increasing metallicity. Since we are assuming that the total heavy-element abundance in the Galactic disk has monotonically increased over the past five billion years [19, 20], i. e., that metallicity is a statistical indicator of age, field stars of this subsystem should display a decrease in the relative abundances of $r$-elements with increasing metallicity, with this decrease being the same as that for the primary $\alpha$-elements, since both types of element are produced in Type II supernovae.

The metallicity dependences of the relative abundances of europium — the only $r$-element studied — is presented in Fig. 1e. The field dwarfs and giants form a single sequence, with their dependences fully coincident. The regression fit constructed for all open clusters also coincides within the uncertainties (this fit is not shown in the figure), but the scatter in the $[\mathrm{Eu} / \mathrm{Fe}]$ values is appreciably higher for the clusters than for the field stars. The europium abundances for most of the clusters were determined in only one study, but using several stars (the Eu abundances for only 12 clusters have been determined in multiple studies). Clusters that deviate considerably from the band formed by the field giants are denoted in various fonts in Fig. 1e. Clusters in which the Eu abundance was determined in several studies or using more than five stars in a single study are denoted in bold font, those in which this abundance was determined using from two to four stars in a single study in regular font, and those in which it was determined using only one star in a single study in italic. Clusters whose Eu abundances were determined using only one star lie at the periphery of the cluster of points in this diagram. However, the position of the regression fit obtained after excluding these clusters remains virtually unchanged.

Different groups of clusters. Let us now compare the elemental abundances for clusters displaying different kinematics. We showed in $[3,4]$ that the population of open clusters includes two groups with different kinematics, as well as different chemical and physical properties. We separated the two groups based on the orbital parameter proposed in [21], $\left(Z_{\max }^{2}+4 e^{2}\right)^{1 / 2}$, with the division being 0.40 (for more refined information about this boundary value, see [5]). (Here, e is the eccentricity of the Galactic orbit and $Z_{\max }$ the maximum distance of points on the orbit from the Galactic plane in kiloparsec.) We referred to clusters with low orbital parameters as "Galactic" and to those with high orbital parameters as "peculiar". We also placed clusters with thin disk metallicities that were uncharacteristically low for field stars $([\mathrm{Fe} / \mathrm{H}] \approx<-0.2)$ in the peculiar group. However, there are no clusters with low, circular orbits that are simultaneously metal-poor in our sample of 90 clusters, since all such clusters are distant and too young to contain giants that could be used for spectroscopic determinations of their elemental abundances. Here, all the clusters with low orbital parameters are metal-rich (and they are Galactic), while those with high orbital parameters are peculiar.

The peculiar clusters are shown by hollow circles and the Galactic clusters by circled points in Fig. 1. The behavior of the $s$-element relative abundances in the first four panels of this figure do not display any significant differences between these groups of clusters. Both groups have equally large scatters in the $[s / \mathrm{Fe}]$ values and, on average, equally large enhancements of these values over those for the field stars. The $r$-element relative abundances for the different groups of clusters, shown in Fig. 1e, behave quite differently. The Galactic clusters lie mainly in the band occupied by field stars (the Eu abundance for NGC 2632 was determined for five stars in a single study, and the Eu abundance for Mel 111 was determine using only one star in a single study).

It is striking that the $[\mathrm{Eu} / \mathrm{Fe}]$ values for all the peculiar clusters lie below the regression fit for the 
field stars (which is the mean line for these stars) in the region $[\mathrm{Fe} / \mathrm{H}]>-0.1$. The Eu abundances for all these clusters were determined using several stars, sometimes from several studies, making them fairly trustworthy. The metal-poor clusters, which mainly belong to the peculiar group, occupy a range of $[\mathrm{Eu} / \mathrm{Fe}]$ that is symmetrically broader than the band for the field stars. Some clusters have very low and others very high $[\mathrm{Eu} / \mathrm{Fe}]$ values, not characteristic for field stars. Note, however, that the parameters for the three clusters Be 31, NGC 2266, and NGC 2158, which display the strongest deviations from the mean $[\mathrm{Eu} / \mathrm{Fe}]$, were determined from only one star in a single study. The Eu abundances of the remaining clusters lying far from the mean values were determined using several stars. As a result, the linear regression fit for clusters with high, elongated orbits has an appreciably more negative slope than the slope for the field stars $\left(\left(r=-0.70 \pm 0.03\right.\right.$, with $\left.P_{N} \ll 1 \%\right)$. This slope remains unchanged if we exclude clusters with less trustworthy $[\mathrm{Eu} / \mathrm{Fe}]$ values.

We showed in $[5]$ that metal-poor clusters $([\mathrm{Fe} / \mathrm{H}]<-0.1)$ with high, elongated orbits have lower relative abundances of primary $\alpha$-elements (oxygen and magnesium) than do field stars of the Galactic thin disk. One possible explanation is that the star-formation rate in the interstellar matter far from the Galactic plane from which the high-velocity clouds stimulating the birth of these clusters is lower than the typical rate in the thin disk. In this case, the relative abundances of Eu should also be reduced, since $r$-elements are also ejected in Type II supernovae. This difference could also come about if the masses of the Type II supernovae that enriched the medium from which the clusters formed were lower, since the output of primary $\alpha$-elements decreases with decreasing mass of the presupernova. However, the relative abundances of Eu in such clusters should then be enhanced relative to those in field stars of the same metallicity, since $r$-elements are produced in supernova outbursts of less massive stars $\left(8-10 M_{\odot}\right)$, than those producing $\alpha$-elements $\left(>10 M_{\odot}\right)$. The large scatter in the $[\mathrm{Eu} / \mathrm{Fe}]$ values in Fig. 1e for metal-poor clusters with high, elongated orbits could testify to the joint action of both of these effects.

The $[\mathrm{O}, \mathrm{Mg} / \mathrm{Eu}]$ ratio is a more sensitive indicator of the masses of Type II supernovae. We therefore show in Fig. If a plot of metallicity versus the abundance of primary $\alpha$-elements relative to $\mathrm{Eu}$. The regions occupied by the field giants and dwarfs also coincide in this diagram. Clusters that lie far from the region occupied by field stars are indicated. Only three of these have their Eu abundances determined from only one star, while the rest have $\mathrm{Eu}$ abundances determined from several stars. The abundances of primary $\alpha$-elements for all the clusters except for Be 31 were likewise determined using several stars. It is clear that the linear regression fits for clusters with high, elongated orbits and for field stars differ outside the uncertainties. Excluding the most discrepant points in the diagram does not reduce the slope for the peculiar clusters.

The $[\mathrm{O}, \mathrm{Mg} / \mathrm{Eu}]$ ratios for the metal-poor clusters lie on either side of the mean line for the field stars, and both of the mechanisms noted above could be responsible for their large scatter, as well as possible systematic errors in the abundances determined in various studies. In addition, a contribution could be made by inhomogeneity of the medium from which the high-velocity clouds formed, and uncertainty in the fraction of matter they contribute during the formation of the clusters. In the positive direction, the $[\mathrm{O}, \mathrm{Mg} / \mathrm{Eu}]$ ratios of the clusters do not extend beyond the band occupied by the field stars, suggesting these clusters formed from medium with an admixture of matter ejected from supernovae with the same masses as those exploding in the thin disk. However, the $[\mathrm{O}, \mathrm{Mg} / \mathrm{Eu}]$ ratios deviate much more in the negative direction in Fig. 1f, with the abundances of all elements for the most discrepant clusters (NGC 2243, Tombaugh 2, and Melotte 71) being determined from several stars, making them fairly reliable. This means that the most likely origin for these deviations is a lower mass for the Type II supernovae that enriched the matter from which these clusters formed, compared to their masses in the plane of the disk. At the same time, all the metal-rich clusters with high, elongated orbits display not only low values of $[\mathrm{Eu} / \mathrm{Fe}]$, but high values of $[\mathrm{O}, \mathrm{Mg} / \mathrm{Eu}]$ as well. This likely testifies that such clusters formed from mixed material that was enriched by Type II supernovae with very high masses. 


\section{AGE DEPENDENCES OF THE RELATIVE ABUNDANCES OF NEUTRON-CAPTURE ELEMENTS}

$s$-Elements. The age dependences of the relative $s$-element abundances for the clusters and field giants behave very differently (Fig. 2a-2d). Recall that we estimated the ages of the red giants from their masses, determined from models for their atmospheres. The fact that the age-metallicity relations for our red giants [5, Fig. 3a] and field dwarfs [20] essentially coincide confirms that the ages of the red giants are not subject to appreciable systematic errors associated with uncertainty in the modeling. The ages of the dwarfs were obtained using two independent methods: from theoretical isochrones and from their chromospheric activity.

We can see that the metallicity in the Galactic disk was essentially independent of age during the first several billion years, while it began to monotonically increase in the last four to five billion years. Because it was possible to calculate only a lower limit for the ages of giants whose masses were given in the initial sources with accuracy to only one significant figure after the decimal point, the diagram constructed using these values is discrete, and contains nine age intervals. The corresponding step is several billions of years for high ages, i. e., for low masses, and decreases to tens of millions of years for the youngest giants. It was easiest to compare the behavior of the age dependences of [el/Fe] for the open clusters and field giants by approximating the latter using line segments joining the mean ratios $\langle[\mathrm{el} / \mathrm{Fe}]\rangle$ in these nine age intervals, and approximating the behavior for the clusters using a smoothed trend obtained using a sliding average.

All the plots in Fig. 2 clearly show a substantial excess of $\langle[\mathrm{el} / \mathrm{Fe}]\rangle$ for the clusters compared to the field giants (the smallest excess is that for yttrium). Although none of the correlations for the field giants are formally signficant, all the ratios [el/Fe] (apart from $[\mathrm{Ba} / \mathrm{Fe}]$ ) overall seem to increase slightly with age. On the contrary, the relative abundances for yttrium and cerium for the clusters show significant anti-correlations (in both cases, $P_{N}<1 \%$ ). Weak tendencies for a decrease with age for the clusters are also displayed by barium and lanthanum, but the correlations for these elements are formally not significant (in both cases, $P_{N}>15 \%$ ). (The fitted regression lines are not included in the graphs, to avoid cluttering the figures.) At low ages, all the $s$-element abundances for the clusters display $[s / \mathrm{Fe}]$ values significantly higher than the solar values, while all these ratios are close to the solar values at higher ages (the ratios for yttrium and cerium are even slightly lower than solar). The mean relative abundances of all the $s$-elements for the field giants apart from barium are significantly lower than solar at low ages, while they are solar within the uncertainties at higher ages. The mean values $\langle[\mathrm{Ba} / \mathrm{Fe}]\rangle$ for the field giants are equal to zero within the uncertainties at all ages (the relative abundances of barium are higher than those of the remaining $s$-elements for both the field giants and the open clusters).

Comparing the relative abundances of yttrium and lanthanum in stars in young clusters and in field stars of the thin disk, Mishenina et al. $[9,10]$ concluded that the age dependences of $[\mathrm{Y} / \mathrm{Fe}]$ and $[\mathrm{La} / \mathrm{Fe}]$ for these two types of objects were similar. They took observed differences between the young objects within $\delta[\mathrm{Y} / \mathrm{Fe}], \delta[\mathrm{La} / \mathrm{Fe}] \approx 0.1$ to be insignificant, and suggested the appreciable excesses in $[\mathrm{La} / \mathrm{Fe}]$ observed for some clusters were due to differences between the values obtained in different studies, or the possible influence of an age variation of the metallicity resulting from the iron output from Type Ia supernovae. They also note that a final resolution of this question requires additional study.

Our yttrium abundances for 34 clusters younger than one billion years averaged over many studies yield $\langle[\mathrm{Y} / \mathrm{Fe}]\rangle=+0.07 \pm 0.02$, while 75 field giants of the same age display $\langle[\mathrm{Y} / \mathrm{Fe}]\rangle=-0.06 \pm$ 0.01. Lanthanum did not show any age dependence (Fig. 2c); however, overall, the clusters with La abundances have $\langle[\mathrm{La} / \mathrm{Fe}]\rangle=+0.12 \pm 0.02$, while the field red giants have $\langle[\mathrm{La} / \mathrm{Fe}]\rangle=-0.13 \pm 0.01$. In other words, there is an obvious difference in these mean values that lies far outside the uncertainties, making them statistically significant and impossible to ignore. Mishenina et al. [9] noted the relative abundances of barium in clusters to be enhanced relative to field stars, especially at low ages, in full 
consistency with our results.

$r$-elements. The age dependences of the relative abundance of the $r$-element Eu for the clusters and field giants behave somewhat differently than the ratio $[\alpha / \mathrm{Fe}]$, although both elements are ejected in Type II supernovae. Figure $2 \mathrm{e}$ shows that the mean $[\mathrm{Eu} / \mathrm{Fe}]$ ratios for the two types of object do not depend on age for ages $<2$ billion years, and have $\langle[\mathrm{Eu} / \mathrm{Fe}]\rangle \approx 0.07$, slightly higher than the solar values. At higher ages, the mean ratios for the field giants begin to grow to $\approx 4.5$ billion years, after which they remain constant. (Although this growth is modest, the correlation coefficient for the $[\mathrm{Eu} / \mathrm{Fe}]-$ age dependence differs from zero beyond the uncertainties.) The mean ratios $\langle[\mathrm{Eu} / \mathrm{Fe}]\rangle$ for the clusters are not significant, but systematically decrease, becoming lower than those for the field giants at ages $>2$ billion years.

Figure $2 \mathrm{f}$ presents age dependences for the $[\mathrm{O}, \mathrm{Mg} / \mathrm{Eu}]$ ratios for these same objects. The ratios for the field stars smoothly increase until about three billion years, then cease to vary with age. The mean line for all the clusters behaves roughly similarly. All the Galactic clusters (apart from two located very low in the diagram) are located in the band occupied by the field stars. Supposing that the primary $\alpha$-elements and $r$-elements are produced in Type II supernovae with different masses, the increase in $[\mathrm{O}, \mathrm{Mg} / \mathrm{Eu}]$ with age for the field giants suggests that the masses of Type II supernovae were higher in the initial stages of the formation of the thin disk than at the current epoch.

The metal-poor clusters with high, elongated orbits can be divided into two groups. One has [O, $\mathrm{Mg} / \mathrm{Eu}]$ ratios that increase with age slightly more steeply than the field stars, with their sequence lying below the sequence for the field giants. The other, which contains five clusters with ages in the range $0.5-3$ billion years, has higher $[\mathrm{O}, \mathrm{Mg} / \mathrm{Eu}]$ ratios than field stars of the same age. In other words, it appears that the metal-poor group of clusters with high, elongated orbits is made up of two subgroups. The metal-rich clusters with high, elongated orbits include representatives of all ages; half are located in the band occupied by the field stars, while the others are located in the uppermost region in the figure.

\section{RELATIONSHIP BETWEEN THE RELATIVE ABUNDANCES OF NEUTRON-CAPTURE ELEMENTS AND THE SPATIAL POSITIONS AND ELEMENTS OF THE GALACTIC ORBITS}

The abundances of essentially all elements in field stars of the Galactic thin disk decrease with increasing Galactocentric distance. (The barium abundance is an exception, as it does not display any radial gradient in Cepheids [22].) In [5], we considered the radial and vertical gradients of the metallicity and $[\alpha / \mathrm{Fe}]$ ratios for clusters and field stars, which showed differences between these two types of object. Figure 3a presents dependences of $[r / \mathrm{Fe}]([\mathrm{Eu} / \mathrm{Fe}])$ on Galactocentric position for the open clusters, Cepheids, and field giants. (We used the apogalactic radii of their orbits $R_{a}$ for the field giants, since they are all currently located near the Sun.) This diagram shows that the relative abundances of $r$-elements in field stars and clusters increase with increasing Galactocentric distance (the correlation coefficients for both types of field stars differ from zero beyond the uncertainties, while the correlation for the clusters is not significant due to the large scatter in the $[\mathrm{Eu} / \mathrm{Fe}]$ values, $P_{N} \approx 27 \%$ ).

A negative metallicity gradient arises for field stars of the thin disk because of the decrease in the star-forming rate with distance from the Galactic center [19]. The iron abundance in the interstellar medium increases with time when the star-forming rate decreases in a stellar-gas system, mainly due to ejection from comparatively long-lived progenitors of Type Ia supernovae. Therefore, like $[\alpha / \mathrm{Fe}]$, $[r / \mathrm{Fe}]$ should increase with increasing $R_{G}$, both currently and in subsequent generations of stars, as is observed for Eu in Fig. 3a (and for $[\alpha / \mathrm{Fe}]$ in Fig. $4 \mathrm{~b}$ of [5]). On the other hand, open clusters (especially those with high, elongated orbits) are genetically related to each other only weakly, and radial gradients can arise in them only if they acquired at the protocloud phase an admixture of matter 
with a different chemical composition, which simultaneously conveyed an impulse that elongated their initially circular orbits. The existence of a correlation between the metallicity and distance from the Galactic center for the clusters testifies that the amount of admixed metal-poor matter they acquired from high-velocity clouds is proportional to the associated impulse acquired [5].

We found in [5] that $\alpha$-elements in clusters display a slightly lower slope in the dependence of $[\alpha / \mathrm{Fe}]$ on $R_{G}$ (see Fig. 4b of [5]) than field giants and Cepheids. At the same time, the radial gradients of the relative abundances of Eu for the clusters, field Cepheids, and field giants in Fig. 3a are very similar. However, corresponding regression fits for the peculiar clusters in Fig. 3a display a significantly higher slope than the slope for both types of field stars. Thus, the Galactocentricdistance dependences for the relative abundances of $\mathrm{Eu}$ for the peculiar clusters with high, elongated orbits and the field stars are substantially different.

In order to correct for possible systematic errors in the abundances for each element, we further consider the behavior of averaged abundances for two $s$-elements (Ba and La). (Unfortunately, the abundances of the different $s$-elements were determined for stars in different clusters, and we therefore used mean ratios for these two $s$-elements for all the objects, since they were most often determined for the same clusters.) The relative abundances of $s$-elements in Fig. $3 \mathrm{~b}$ show that the radial gradients obtained for the open clusters based on their current positions are much greater, and the sequence of values itself much higher, than for the field giants, based on the apogalactic radii of their orbits. The slope of the $[s / \mathrm{Fe}]-R_{a}$ relation does not change if we adopt the apogalactic radii of the cluster orbits instead of their current positions. (On the other hand, for reasons we do not currently understand, the $[s / \mathrm{Fe}]-R_{G}$ relation and associated gradients for the clusters and field Cepheids proved to be very similar.) All the corresponding correlations are significant $\left(P_{N}<5 \%\right)$.

The output of $s$-elements depends on the metallicity of the stars that eject them, and is not related to the history of star formation in the stellar-gas system. Therefore, the presence of the observed slopes of the $[s / \mathrm{Fe}]$ dependences on the Galactocentric positions for the field giants in Fig. 3b can be explained by the existence of a negative radial metallicity gradient in the Galactic disk, while we suggest that the cluster dependence is due to the relationship between the amount of metal-poor matter they acquired and the velocity impulse imparted to them by the high-velocity clouds that stimulated their birth.

Figures 3c, 3d present the dependences of the relative abundances of the $r$ and $s$ elements in clusters on the orbital parameter $\left(Z_{\max }^{2}+4 e^{2}\right)^{1 / 2}$. (Five clusters with implausibly high orbital parameters, $>3.0$, which were probably a result of errors in their spatial velocities, were excluded.) The lines in all the panels show the regression lines for peculiar clusters with $\left(Z_{\max }^{2}+4 e^{2}\right)^{1 / 2}>0.40$. These plots show that the dependences of the abundances of all elements considered on this kinematic parameter are formed by the clusters with high, elongated orbits. As our analysis showed, the points in the orbit that are maximally distant from the Galactic plane $Z_{\max }$ make a large contribution to the orbital parameter, and not the orbital eccentricity. This figure shows that the slopes of the dependences for the field giants and clusters coincide within the uncertainties in both diagrams; while the relative abundances of Eu for the clusters are, on average, lower for any orbital parameters, the $[s / \mathrm{Fe}]$ ratios are much higher.

\section{DISCUSSION}

According to [4], only Galactic clusters - metal-rich clusters $([\mathrm{Fe} / \mathrm{H}]>\approx-0.2)$ with flat, circular orbits $\left(\left(Z_{\max }^{2}+4 e^{2}\right)^{1 / 2}<0.40\right)$ - could form from the same material as field stars. Therefore, it would seem that the relative abundances of their neutron-capture elements should lie in the band occupied by field stars in plots of the metallicity dependences of these abundances. We showed in [5] that the relative abundances of $\alpha$-elements satisfy this hypothesis. This is also more or less true for the relative abundances of the $r$-element Eu (Fig. 1e). However, the relative abundances of $s$-elements in Galactic clusters demonstrate substantial excesses relative to field stars.

The dependences for all the neutron-capture elements for clusters with high, strongly elongated 
orbits differ from those for field stars. Since $r$-elements and primary $\alpha$-elements are ejected into the interstellar medium in Type II supernovae, this suggests that they should display the same metallicity dependences. In reality, the slopes of plots of $[\mathrm{Eu} / \mathrm{Fe}]$ versus $[\mathrm{Fe} / \mathrm{H}]$ for peculiar clusters are significantly higher than those for field stars (Fig. 1e), while the fitted regression line for the relative abundances of primary $\alpha$-elements for clusters [5, Fig. 2c] has an appreciably lower slope than the dependence for the field giants. Thus, metal-rich and metal-poor clusters with high, elongated orbits display differences from field giants with the same metallicity in both $[\mathrm{O}, \mathrm{Mg} / \mathrm{Fe}]$ and $[\mathrm{Eu} / \mathrm{Fe}]$. The $[\mathrm{O}, \mathrm{Mg} / \mathrm{Eu}]$ ratios for these two groups of clusters with high, elongated orbits also proved to be very different. All the metal-rich clusters have higher values of these ratios than field giants, on average. The metal-poor clusters display enhanced and reduced ratios of primary $\alpha$-elements to Eu (although remaining within the region occupied by field giants); however, among the latter, several clusters deviate far behond the sequence of the field stars.

The age dependences of the relative abundances of the $r$-element $\mathrm{Eu}$ in clusters and field stars also appear very different: $[\mathrm{Eu} / \mathrm{Fe}]$ (like $[\alpha / \mathrm{Fe}]$ ) increases with age for the field giants, while these ratios monotonically decrease with age for the clusters (Fig. 2e). Although $[\alpha / \mathrm{Fe}]$ increases with age for the clusters, this increase is appreciably slower than the increase for field stars. As a result, the mean relative abundances of both $\alpha$-elements and Eu for clusters and field stars coincide at low ages; at high ages, the $[\alpha / \mathrm{Fe}]$ and $[\mathrm{Eu} / \mathrm{Fe}]$ ratios for clusters become appreciably lower.

At all Galactocentric distances, a substantial fraction of clusters with high, elongated orbits have $[\mathrm{Eu} / \mathrm{Fe}]$ values lying below the band occupied by the field stars (Fig 3c). The [Eu/Fe] dependence on the orbital parameter also lies far lower for clusters with high, elongated orbits than for field giants, and the two regression slopes differ significantly. As a result, the relative abundances of Eu are different for clusters with high, elongated orbits and field stars. The differences between metal-rich and metal-poor peculiar clusters are also clear. In particular, the more metal-poor group has higher $\langle[\mathrm{Eu} / \mathrm{Fe}]\rangle$ values, on average, corresponding to an appreciable slope in a plot of $[\mathrm{Fe} / \mathrm{H}]$ versus $[\mathrm{Eu} / \mathrm{Fe}]$. This same difference in the mean relative abundances of Eu led to the existence of a substantial radial gradient for the peculiar clusters, and a dependence on the orbital parameter.

The properties of the relative abundances of $s$-elements in open clusters and field stars of the thin disk also differ substantially. Although the $[s / \mathrm{Fe}]$ ratios for both types of object decrease with increasing $[\mathrm{Fe} / \mathrm{H}]$ in approximately the same way, the cluster values are substantially higher than those for the field stars for any metallicity (Fig. 1). The $[s / \mathrm{Fe}]$ ratios for the field Cepheids are similarly high. We proposed in [14] that the high relative abundances of $s$-elements in young Cepheids could have come about because collapsing high-mass supernovae ceased to explode in the thin disk several hundreds of millions of years ago, as a result of an increase in the overall metallicity. As a result, the relative abundances of elements ejected by Type II supernova were redistributed in the interstellar medium. However,this explanation is not suitable for clusters, since many of the clusters for which elemental abundances are available have high ages, sometimes comparable to the age of the Galactic thin disk, and relative abundances of $s$-elements that are high for a given metallicity are also displayed by clusters with fairly low $[\mathrm{Fe} / \mathrm{H}]$ values.

In contrast to $\alpha$-elements and $r$-elements, $s$-elements display high relative abundances compared to field stars at ages $\leq 2$ billion years, while their mean values become similar at high ages (Fig. 2). $[s / \mathrm{Fe}]$ increases with increasing Galactocentric distance for clusters, with the dependences for the clusters and field Cepheids fully coinciding. The radial gradient in the relative abundances of $s$ elements is slightly smaller for field giants, and the sequence itself lies much lower (Fig 3b).

The $[s / \mathrm{Fe}]$ ratio for clusters also increases with the orbital parameter, while this ratio is essentially independent of the shape of the orbit for the field stars. As a result, these elements likewise testify to the absence of a genetic relationship between field stars of the thin disk and open clusters. It was concluded in $[9,10]$, where the same method was used to obtain the relative barium abundances in several young open clusters and in field stars, that the origin of the higher $[\mathrm{Ba} / \mathrm{Fe}]$ values for clusters was not associated with systematic errors. It is not yet clear how to explain this result. It appears that the same situation occurs with the relative abundances of the remaining $s$-elements in open 
clusters.

\section{CONCLUSION}

Thus, the characteristic differences in the behavior of the relative abundances of primary $\alpha$-elements and $r$-elements in open clusters support the conclusion, based on an analysis of their metallicities and the elements of their Galactic orbits, that metal-poor clusters $([\mathrm{Fe} / \mathrm{H}]<-0.1)$ with high, elongated orbits were formed during interactions of metal-poor high-velocity clouds and the interstellar material of the Galactic thin disk. The lower relative abundances of primary $\alpha$-elements, and frequently lower $[\mathrm{O}, \mathrm{Mg} / \mathrm{Eu}]$ ratios, in these clusters compared to field stars, can most logically be understood if the masses of the Type II supernovae that enriched the material from which these high-velocity clouds formed more often fell in the range $(8-10) M_{\odot}$, than is the case for the supernova that enriched the material of the thin disk. The large scatter in the relative abundances of Eu in metal-poor clusters with high, elongated orbits suggest that this material was poorly mixed.

On the other hand, the higher $[\mathrm{O}, \mathrm{Mg} / \mathrm{Fe}]$ and $[\mathrm{O}, \mathrm{Mg} / \mathrm{Eu}]$ ratios in clusters with high, elongated orbits and $[\mathrm{Fe} / \mathrm{H}]>-0.1$, compared to field stars, suggests that these clusters were born as a result of interactions of matter of the thin disk with high-velocity clouds formed in a "Galactic fountain" process. Such clouds are believed to form from matter ejected from the thin disk as a result of massive supernova explosions, after which this material falls back toward the disk, stimulating the birth of open clusters in some cases. The detection of higher relative abundances of primary $\alpha$-elements and lower relative abundances of $r$-elements in metal-rich clusters with high, elongated orbits, on average, compared to field stars, apparently testifies that such Galactic fountains form mainly as a result of the explosion of more massive Type II supernovae $\left(M>10 M_{\odot}\right)$, for which the output of primary $\alpha$-elements is higher, and the output of $r$-elements lower, than is the case for less massive supernovae.

A distinguishing property of the relative abundances of $s$-elements in open clusters (independent of the group to which they belong) is their appreciable excesses compared to field stars of the thin disk. Only for very old clusters $(t>4$ billion years) with high, elongated orbits the $[s / \mathrm{Fe}]$ ratios are the same as those for field stars, within the uncertainties, while they are substantially higher in younger clusters, independent of the nature of the clusters. Since most clusters are young, on average, clusters display enhanced relative abundances of all $s$-elements, compared to field stars. This is not inconsistent with the proposed origin of clusters with high, elongated orbits presented above, but it is not possible to place them in a logical, self-consistent scheme. There is no question that there remains only possible differences of their properties from those for the same elements in field stars of the Galactic thin disk. The possible origins of this behavior for the $s$-elements is not yet understood.

\section{ACKNOWLEDGMENTS}

The authors thank N.O. Budanova for help in collecting the material on the chemical compositions of open cluster stars. This work was supported by the Ministry of Education and Science of the Russian Federation (state contracts 3.961.2014/K, 213.01-11/2014-5 (project code 26.63)), and the Southern Federal University (grant 213.01-2014/013-VG).

[1] Z. -Yu. Wu, X. Zhou, J. Ma, and C.-H. Du, Monthly Not. Roy. Astron. Soc. 399, 2146 (2009)

[2] D. Vande Putte, T.P. Garnier, I. Ferreras, R.P. Mignani, and M. Cropper, Monthly Not. Roy. Astron. Soc. 407, 2109 (2010)

[3] M.L. Gozha, T.V. Borkova, and V.A. Marsakov, Astron. Lett. 38, 506 (2012).

[4] M. L. Gozha, V. V. Koval', and V. A. Marsakov, Astron. Lett. 38, 519 (2012). 
[5] V.A. Marsakov, M.L. Gozha, V.V. Koval', and L.V. Shpigel', Astron. Rep. 60, 43 (2016)

[6] V. D’Orazi, L. Magrini, S. Randich, D. Galli, M. Busso, and P. Sestito, Astrophys. J. 693, L31 (2009)

[7] E. Maiorca, S. Randich, M. Busso, L. Magrini, and S. Palmerini, Astrophys. J. 736, 120 (2011)

[8] A. Serminato, R. Gallino, C. Travaglio, S. Bisterzo, and O. Straniero, Publ. Astron. Soc. Australia 26, 153 (2009)

[9] T.V. Mishenina, M. Pignatary, S.A. Korotin, C. Soubiran, C. Charbonnel, F.K. Thielemann, T.I. Gorbaneva, and N.Yu. Basak, Astron. and Astrophys. 552, 128 (2013)

[10] T. Mishenina, M. Pignatari, G. Carraro, V. Kovtyukh, L. Monaco, S. Korotin, E. Shereta, I. Yegorova, and F. Herwig, Monthly Not. Roy. Astron. Soc. 446, 3651 (2015)

[11] H.R. Jacobson and E.D. Friel, Astron. J. 145, 107 (2013)

[12] D. Yong, B.W. Carney, and E.D. Friel, Astron. J. 144, 95 (2012).

[13] V. D’Orazi, K. Biazzo, S. Desidera, E. Covino, S.M. Andrievsky, and R.G. Gratton, Monthly Not. Roy. Astron. Soc. 423, 2789 (2012)

[14] V.A. Marsakov, V.V. Koval', V.V. Kovtyukh, and T.V. Mishenina, Astron. Lett. 39, 851 (2013)

[15] S. Bisterzo, R. Gallino, O. Straniero, S. Cristallo, and F. Käppeler, Monthly Not. Roy. Astron. Soc. 418, 284 (2011)

[16] S.E. Woosley, J.R. Wilson, G.J. Mathews, R.D. Hoffman, and B.S. Meyer, Astrophys. J. 433, 229 (1994)

[17] M. Pignatari, R. Gallino, M. Heil, M. Wiescher, F. Käppeler, F. Herwig, and S. Bisterzo, Astrophys. J. 710, 1557 (2010)

[18] F.-K. Thielemann, K. Nomoto, and Y. Yokio, Astron. and Astrophys. 158, 17 (1986)

[19] V.A. Marsakov and T.V. Borkova, Astron. Lett. 32, 376 (2006)

[20] V.A. Marsakov, V.V. Koval', T.V. Borkova, and M.V. Shapovalov, Astron. Rep. 55, 667 (2011).

[21] R.G. Gratton, E. Carretta, R. Claudi, S. Lucatello, and M. Barbieri, Astron. and Astrophys. 404, 187 (2003)

[22] S.M. Andrievsky, J.R.D. Lepine, S.A. Korotin, R.E. Luck, V.V. Kovtyukh, and W.J. Maciel, Monthly Not. Roy. Astron. Soc. 428, 3252 (2013) 

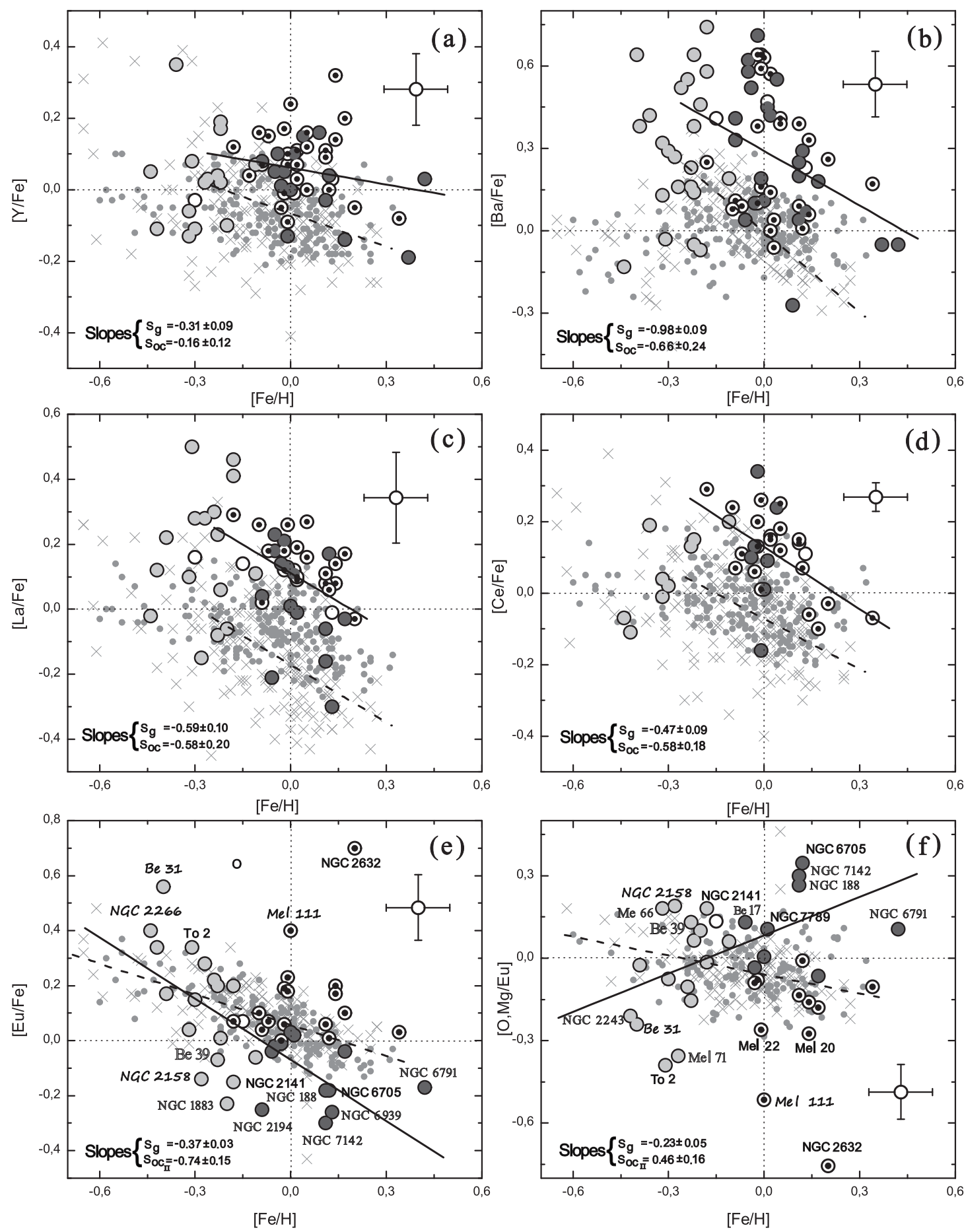

Figure 1: Metallicity dependences of the relative abundances of (a)-(d) four $s$-elements, (e) an $r$ element, and (f) $[\mathrm{O}, \mathrm{Mg} / \mathrm{Eu}]$ in open clusters (circles), field red giants (x's), and field dwarfs (gray points). The circled dots show clusters with low, circular orbits (orbital parameter $\left(\left(Z_{\text {max }}^{2}+4 e^{2}\right)^{1 / 2}<\right.$ 0.40 and $|z|<400 \mathrm{pc})$. The gray circles show clusters in high, elongated orbits, with light gray circles corresponding to metal-poor $([\mathrm{Fe} / \mathrm{H}]<-0.1)$ and dark gray circles to metal-rich clusters. The hollow circles show unclassified clusters. The solid and dashed lines in panels (a)-(d) show the results of regression fits for the clusters and giants, respectively, and the solid and dashed lines in panels (e)-(f) for clusters with high, elongated orbits and field giants, respectively. The slopes for the regression fits are shown in the panels with their errors. The bars show the characteristic uncertainties for all the elements. The dotted lines parallel to the coordinate axes are drawn through the solar values. The names of clusters that deviate strongly from the region occupied by field giants are indicated in panels (e) and (f); those in bold font have oxygen,magnesium, and europium abundance determinations in several studies or for more than five stars in a single study, those in regular font have them for two 

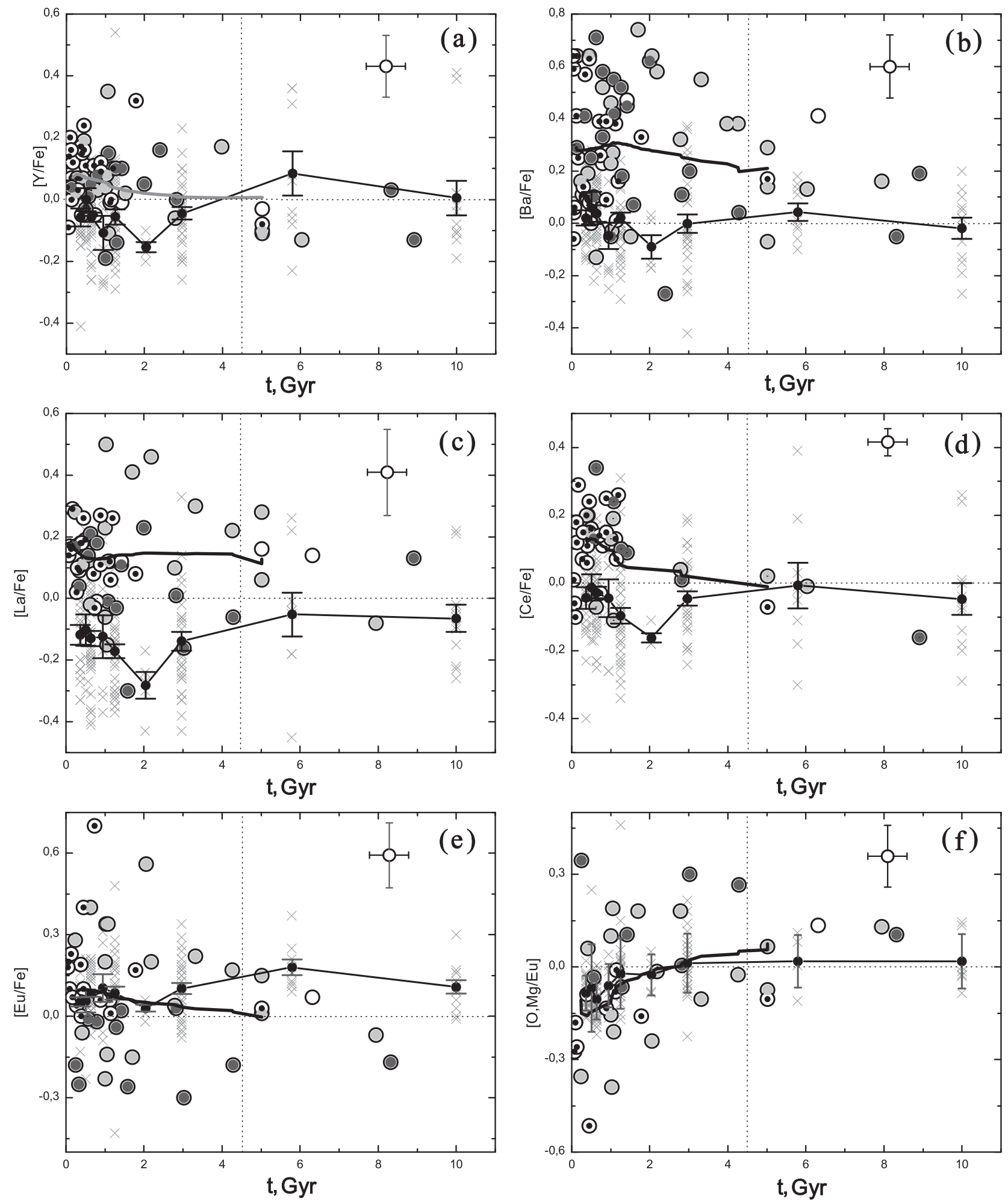

Figure 2: Age dependences of the relative abundances of (a)-(d) four $s$-elements, (e) one $r$-element, and (f) $[\mathrm{O}, \mathrm{Mg} / \mathrm{Eu}]$ for open clusters (circles) and field red giants (x's). The smooth curves show fits to the smoothed trends obtained using a sliding average of the age dependences of the clusters; the curves with jointed line segments show the mean metallicities and $\langle[s / \mathrm{Fe}]\rangle$ values in nine age ranges for the field giants. The bars indicate the mean errors for the giants. The remaining notation is as in Fig. 1. 

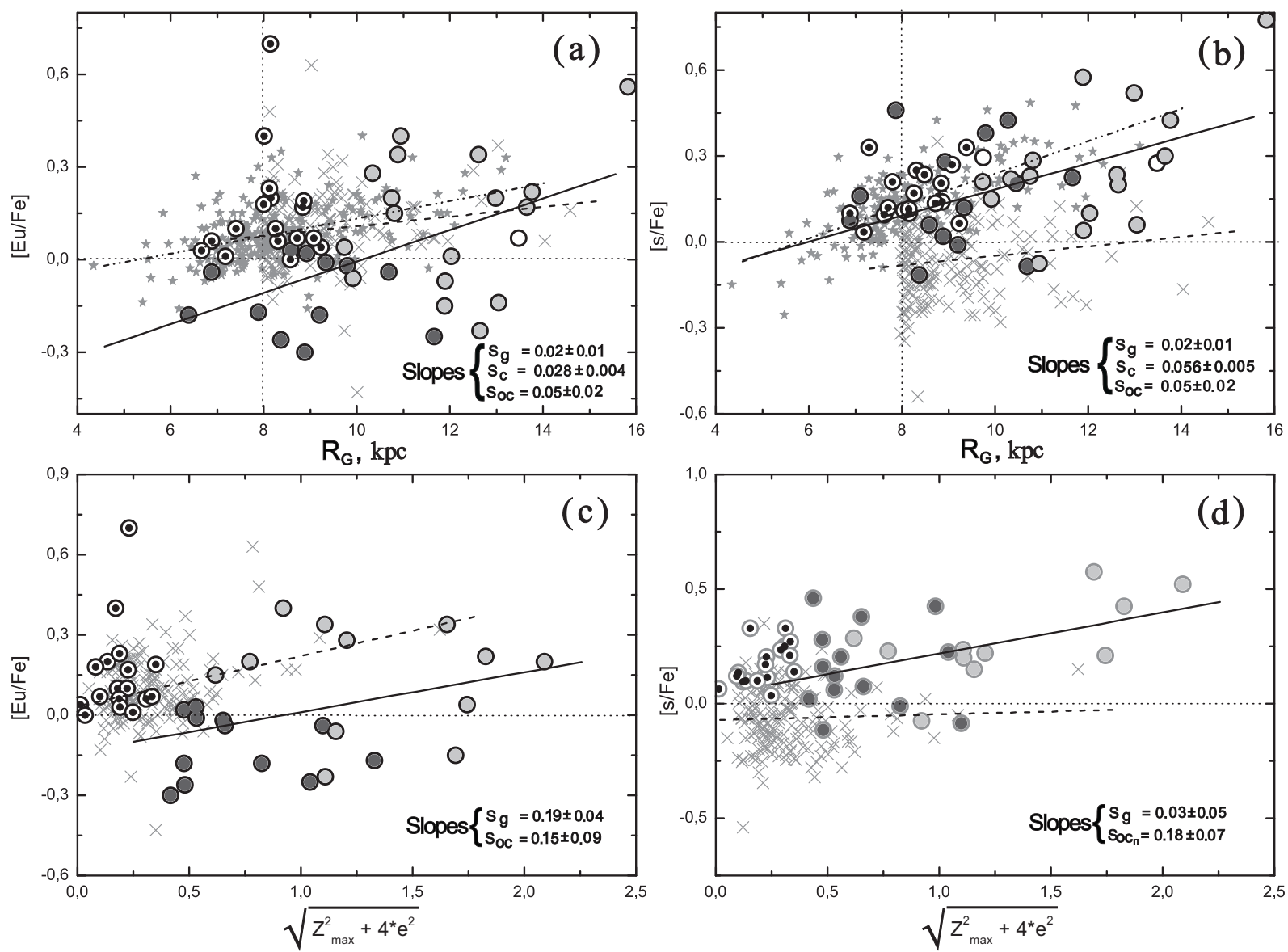

Figure 3: Dependences of the relative abundances of an $r$-element $(\mathrm{Eu})$ and two averaged $s$-elements (La, Ba) on (a)-(b) Galactocentric position and (c)-(d) the orbital parameter $\left(Z_{\text {max }}^{2}+4 e^{2}\right)^{1 / 2}$ for open clusters (circles), field red giants (x's), and field Cepheids (stars). In view of the nearness of the field giants to the Sun, we used the apogalactic radii of their orbits in these plots. The solid, dashed, and dot-dashed lines show the regression fits for the peculiar clusters, field giants, and field Cepheids, respectively. The remaining notation is as in Fig. 1. 